\title{
Finite-Time Chaos Control of a Complex Permanent Magnet Synchronous Motor System
}

\author{
Xiaobing Zhou, ${ }^{1}$ Murong Jiang, ${ }^{1}$ and Xiaomei Cai ${ }^{2}$ \\ ${ }^{1}$ School of Information Science and Engineering, Yunnan University, Kunming 650091, China \\ ${ }^{2}$ Bureau of Asset Management, Yunnan University, Kunming 650091, China \\ Correspondence should be addressed to Xiaobing Zhou; zhouxb.cn@gmail.com
}

Received 21 January 2014; Revised 7 February 2014; Accepted 7 February 2014; Published 12 March 2014

Academic Editor: Hui Zhang

Copyright (c) 2014 Xiaobing Zhou et al. This is an open access article distributed under the Creative Commons Attribution License, which permits unrestricted use, distribution, and reproduction in any medium, provided the original work is properly cited.

\begin{abstract}
This paper investigates the finite-time chaos control of a permanent magnet synchronous motor system with complex variables. Based on the finite-time stability theory, two control strategies are proposed to realize stabilization of the complex permanent magnet synchronous motor system in a finite time. Two numerical simulations have been conducted to demonstrate the validity and feasibility of the theoretical analysis.
\end{abstract}

\section{Introduction}

Permanent magnet synchronous motors (PMSMs) are widely used in various industrial fields because of their simple structure, high efficiency, high power density, low manufacturing cost, and large torque to inertia ratio [1]. It is well known that the existing mathematical models of PMSMs are nonlinear, multivariable, and strongly coupled; therefore, these systems can exhibit complex behaviors, such as Hopf bifurcations, limit cycles, and even chaos [2]. Furthermore, the operation of PMSMs in industrial environment can be affected by many uncertain factors such as unknown system parameters, external load disturbance, friction force, and unmodeled uncertainties. These uncertain factors can seriously degrade the performance quality of PMSM systems [3-5]. So, it is indispensable to study methods of controlling or suppressing chaos in PMSM systems. Up till now, there are numerous methods to control chaos in PMSM systems [6-15]. In [9], a nonlinear feedback control method was proposed to control the chaos in a PMSM system. Loría [10] achieved both setpoint and tracking output regulation of PMSM systems via a simple linear output feedback controller. Based on synchronization characteristics, a continuous feedback control method was proposed to eliminate chaotic oscillations in a
PMSM system [11]. Hou [12] investigated the guaranteed cost control of chaos problem in a PMSM system via TakagiSugeno fuzzy method approach. Choi [13] proposed a simple adaptive controller design method for a chaotic PMSM system based on the sliding mode control theory.

The existing methods stabilize chaotic systems asymptotically; that is, the trajectories of chaotic systems converge to zero with infinite settling time. However, from the practical engineering point of view, it is more crucial to stabilize chaotic systems in a finite time. Therefore, it is important to consider the problem of finite-time stabilization of chaotic systems. Finite-time control is a very useful technique to achieve faster convergence speed in control systems. In addition, the finite-time control technique has demonstrated better robustness and disturbance rejection properties [14]. In [15], Wei and Zhang presented a nonlinear controller to achieve finite-time chaos control in a PMSM system based on the finite-time stability theory.

Since Fowler et al. [16] generalized the real Lorenz model to a complex one, the complex modeling of phenomena in nature and society has been intensively investigated. The complex systems appear in physics and engineering fields, such as detuned lasers, rotating fluids, disk dynamos, electronic circuits, and particle beam dynamics in high energy 
accelerators [17, 18]. Wang and Zhang proposed a complex PMSM system by modifying cross-coupled term in [19], for complex number voltage and complex number current exist widely in motor systems and it is easier to analyze motor systems with complex systems.

Motivated by the above discussion, in the present paper, we construct controllers to stabilize a complex PMSM system. Based on the finite-time stability theorem, two control strategies are proposed to realize chaos control in a finite time. Numerical simulation results show that the proposed controllers are very effective.

\section{System Descriptions}

A PMSM system in a field-oriented rotor can be described by the following equation [20]:

$$
\begin{gathered}
\frac{d i_{d}}{d t}=\frac{1}{L_{d}}\left(u_{d}-R_{1} i_{d}+\omega L_{q} i_{q}\right), \\
\frac{d i_{q}}{d t}=\frac{1}{L_{q}}\left(u_{q}-R_{1} i_{q}-\omega L_{d} i_{d}+\omega \psi_{r}\right), \\
\frac{d w}{d t}=\frac{1}{J}\left(n_{p} \psi_{r} i_{q}+n_{p}\left(L_{d}-L_{q}\right) i_{d} i_{q}-T_{L}-\beta \omega\right),
\end{gathered}
$$

where $i_{d}, i_{q}$, and $\omega$ are the state variables, which represent currents and motor angular frequency, respectively; $u_{d}$ and $u_{q}$ are the direct-axis stator and quadrature-axis stator voltage components, respectively; $J$ is the polar moment of inertia; $T_{L}$ is the external load torque; $\beta$ is the viscous damping coefficient; $R_{1}$ is the stator winding resistance; $L_{d}$ and $L_{q}$ are the direct-axis stator inductors and quadrature-axis stator inductors, respectively; $\psi_{r}$ is the permanent magnet flux, $n_{p}$ is the number of pole-pairs, and the parameters $L_{d}, L_{q}, J, T_{L}$, $R_{1}, \psi_{r}$, and $\beta$ are all positive. When the air gap is even, and the motor has no load and power outage, then the dimensionless equations of a PMSM system can be modeled by

$$
\begin{aligned}
& \dot{z}_{1}=a\left(z_{2}-z_{1}\right), \\
& \dot{z}_{2}=b z_{1}-z_{2}-z_{1} z_{3}, \\
& \dot{z}_{3}=z_{1} z_{2}-z_{3},
\end{aligned}
$$

where $a$ and $b$ are both positive parameters. If the current in the system (1) is plural and the variables $z_{1}$ and $z_{2}$ in the system (2) are complex numbers, by changing cross-coupled terms $z_{1}$ and $z_{2}$ to conjugate form, a complex PMSM system is constructed as follows [20]:

$$
\begin{aligned}
& \dot{z}_{1}=a\left(z_{2}-z_{1}\right), \\
& \dot{z}_{2}=b z_{1}-z_{2}-z_{1} z_{3}, \\
& \dot{z}_{3}=\frac{1}{2}\left(\bar{z}_{1} z_{2}+z_{1} \bar{z}_{2}\right)-z_{3},
\end{aligned}
$$

where $z_{1}=u_{1}+i u_{2}$ and $z_{2}=u_{3}+i u_{4}$ are complex variables, $i=\sqrt{-1} ; u_{i}(i=1,2,3,4)$ and $z_{3}=u_{5}$ are real variables. $\bar{z}_{1}$ and $\bar{z}_{2}$ are the conjugates of $z_{1}$ and $z_{2}$, respectively, and $a$ and $b$ are positive parameters determining the chaotic behaviors and bifurcations of system (3). When the parameters satisfy $1 \leq a \leq 11,10 \leq b \leq 20$, there is one positive Lyapunov exponent, two zero Lyapunov exponents, and two negative Lyapunov exponents for system (4), which means system (3) is chaotic [20].

\section{Basic Conception of Finite-Time Stability Theory}

Finite-time stability means that the states of the dynamic system converge to a desired target in a finite time.

Definition 1 (see [21]). Consider the nonlinear dynamical system modeled by

$$
\dot{x}=f(x),
$$

where the state variable $x \in R^{n}$. If there exists a constant $T>$ $0(T>0$ may depend on the initial state $x(0))$, such that

$$
\lim _{t \rightarrow T}\|x(t)\|=0
$$

and $\|x(t)\| \equiv 0$, if $t \geq T$, then system (1) is finite-time stable.

Lemma 2 (see [22]). Assume that a continuous, positivedefinite function $V(t)$ satisfies the following differential inequality:

$$
\dot{V}(t) \leq-\alpha V^{\lambda}(t), \quad \forall t \geq t_{0}, \quad V\left(t_{0}\right) \geq 0,
$$

where $\alpha>0$ and $0<\lambda<1$ are constants. Then, for any given $t_{0}, V(t)$ satisfies the following inequality:

$$
\begin{gathered}
V^{1-\lambda}(t) \leq V^{1-\lambda}\left(t_{0}\right)-\alpha(1-\lambda)\left(t-t_{0}\right), \quad t_{0} \leq t \leq t_{1}, \\
V(t) \equiv 0, \quad \forall t \geq t_{1},
\end{gathered}
$$

with $t_{1}$ given by

$$
t_{1}=t_{0}+\frac{V^{1-\lambda}\left(t_{0}\right)}{\alpha(1-\lambda)} .
$$

Lemma 3 (see [23]). For any real number $\alpha_{i}, i=1,2, \ldots, k$ and $0<r<1$, the following inequality holds:

$$
\left(\left|\alpha_{1}\right|+\left|\alpha_{2}\right|+\cdots+\left|\alpha_{k}\right|\right)^{r} \leq\left|\alpha_{1}\right|^{r}+\left|\alpha_{2}\right|^{r}+\cdots+\left|\alpha_{k}\right|^{r} .
$$

\section{Finite-Time Chaos Control of a Complex PMSM System}

In order to control chaotic oscillation in the complex PMSM system (3), we add controllers to system (3) and then the controlled system can be expressed by

$$
\begin{aligned}
& \dot{z}_{1}=a\left(z_{2}-z_{1}\right)+\mu_{1}+i \mu_{2}, \\
& \dot{z}_{2}=b z_{1}-z_{2}-z_{1} z_{3}+\mu_{3}+i \mu_{4}, \\
& \dot{z}_{3}=\frac{1}{2}\left(\bar{z}_{1} z_{2}+z_{1} \bar{z}_{2}\right)-z_{3}+\mu_{5},
\end{aligned}
$$


where $\mu_{i}(i=1,2, \ldots, 5)$ are controllers to be designed to achieve finite-time control.

By separating the real and imaginary parts, we have the following real system:

$$
\begin{aligned}
& \dot{u}_{1}=a\left(u_{3}-u_{1}\right)+\mu_{1}, \\
& \dot{u}_{2}=a\left(u_{4}-u_{2}\right)+\mu_{2}, \\
& \dot{u}_{3}=b u_{1}-u_{3}-u_{1} u_{5}+\mu_{3}, \\
& \dot{u}_{4}=b u_{2}-u_{4}-u_{2} u_{5}+\mu_{4}, \\
& \dot{u}_{5}=u_{1} u_{3}+u_{2} u_{4}-u_{5}+\mu_{5} .
\end{aligned}
$$

Next, we apply the finite-time stability theory to design controllers to globally stabilize the unstable equilibrium $O(0,0,0,0,0)$ in a finite time. Two control strategies are proposed to fulfill this goal.

\section{Control Strategy 1}

Theorem 4. If the controllers are designed as

$$
\begin{aligned}
& \mu_{1}=-a u_{3}-u_{1}^{k}, \\
& \mu_{2}=-a u_{4}-u_{2}^{k}, \\
& \mu_{3}=-b u_{1}-u_{3}^{k}, \\
& \mu_{4}=-b u_{2}-u_{4}^{k}, \\
& \mu_{5}=-u_{5}^{k},
\end{aligned}
$$

where $k=q / p$ is a proper rational number, $p$ and $q$ are positive odd integers, and $p>q$, the chaos in the complex PMSM system (10) will be controlled; that is, the complex PMSM system (10) will be asymptotically stabilized at the equilibrium $\mathrm{O}(0,0,0,0,0)$ in a finite time.

Proof. Construct the following Lyapunov function:

$$
V=\frac{1}{2}\left(u_{1}^{2}+u_{2}^{2}+u_{3}^{2}+u_{4}^{2}+u_{5}^{2}\right)
$$

By differentiating the function $V$ along the trajectories of system (11), we have

$$
\begin{aligned}
\dot{V}= & u_{1} \dot{u}_{1}+u_{2} \dot{u}_{2}+u_{3} \dot{u}_{3}+u_{4} \dot{u}_{4}+u_{5} \dot{u}_{5} \\
= & u_{1}\left[a\left(u_{3}-u_{1}\right)+\mu_{1}\right]+u_{2}\left[a\left(u_{4}-u_{2}\right)+\mu_{2}\right] \\
& +u_{3}\left(b u_{1}-u_{3}-u_{1} u_{5}+\mu_{3}\right) \\
& +u_{4}\left(b u_{2}-u_{4}-u_{2} u_{5}+\mu_{4}\right) \\
& +u_{5}\left(u_{1} u_{3}+u_{2} u_{4}-u_{5}+\mu_{5}\right) .
\end{aligned}
$$

Substituting the controllers (12) into the above equation yields

$$
\begin{aligned}
\dot{V}= & u_{1}\left[a\left(u_{3}-u_{1}\right)-a u_{3}-u_{1}^{k}\right] \\
& +u_{2}\left[a\left(u_{4}-u_{2}\right)-a u_{4}-u_{2}^{k}\right] \\
& +u_{3}\left(b u_{1}-u_{3}-u_{1} u_{5}-b u_{1}-u_{3}^{k}\right) \\
& +u_{4}\left(b u_{2}-u_{4}-u_{2} u_{5}-b u_{2}-u_{4}^{k}\right) \\
& +u_{5}\left(u_{1} u_{3}+u_{2} u_{4}-u_{5}-u_{5}^{k}\right) \\
= & -a u_{1}^{2}-u_{1}^{k+1}-a u_{2}^{2}-u_{2}^{k+1}-u_{3}^{2} \\
& -u_{3}^{k+1}-u_{4}^{2}-u_{4}^{k+1}-u_{5}^{2}-u_{5}^{k+1} \\
\leq & -\left(\frac{1}{2}\right)^{-(k+1) / 2}\left[\left(\frac{1}{2} u_{1}^{2}\right)^{(k+1) / 2}+\left(\frac{1}{2} u_{2}^{2}\right)^{(k+1) / 2}\right. \\
& +\left(\frac{1}{2} u_{3}^{2}\right)^{(k+1) / 2}+\left(\frac{1}{2} u_{4}^{2}\right)^{(k+1) / 2} \\
& \left.+\left(\frac{1}{2} u_{5}^{2}\right)^{(k+1) / 2}\right]
\end{aligned}
$$

In light of Lemma 3, we have

$$
\begin{aligned}
\dot{V} & \leq-\left(\frac{1}{2}\right)^{-(k+1) / 2}\left(\frac{1}{2} u_{1}^{2}+\frac{1}{2} u_{2}^{2}+\frac{1}{2} u_{3}^{2}+\frac{1}{2} u_{4}^{2}+\frac{1}{2} u_{5}^{2}\right)^{(k+1) / 2} \\
& =-\left(\frac{1}{2}\right)^{-(k+1) / 2}(V)^{(k+1) / 2}
\end{aligned}
$$

Then from Lemma 2, the controlled system (11) is finite-time stable. This implies that there exists a $T>0$ such that $u_{i} \equiv$ $0(i=1,2, \ldots, 5)$ if $t \geq T$.

\section{Control Strategy 2}

Theorem 5. If the controllers are designed as

$$
\begin{aligned}
& \mu_{1}=-a u_{3}-u_{1}^{k}, \\
& \mu_{2}=-a u_{4}-u_{2}^{k}, \\
& \mu_{3}=-u_{3}^{k}, \\
& \mu_{4}=-u_{4}^{k}, \\
& \mu_{5}=-u_{5}^{k},
\end{aligned}
$$

where $k=q / p$ is a proper rational number, $p$ and $q$ are positive odd integers, and $p>q$, the chaos in the complex PMSM system (10) will be controlled; that is, the complex PMSM system (10) will be asymptotically stabilized at the equilibrium $\mathrm{O}(0,0,0,0,0)$ in a finite time. 

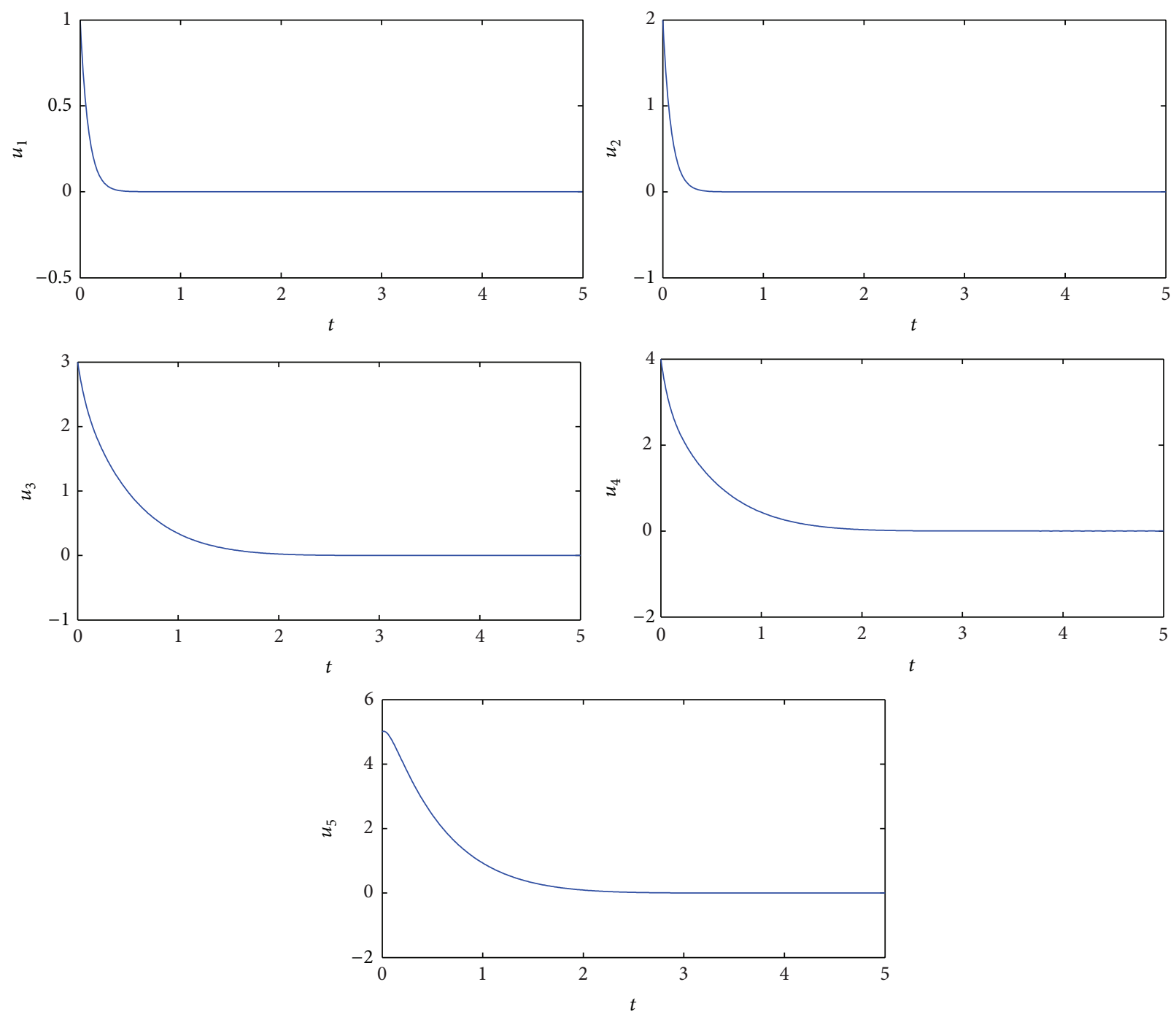

FIGURE 1: The states of the controlled system (11) with controllers (12).

Proof. The design procedure is divided into two steps.

Step 1. Substituting the controllers $\mu_{1}$ and $\mu_{2}$ into the first two parts of system (11) yields

$$
\begin{aligned}
& \dot{u}_{1}=-a u_{1}-u_{1}^{k}, \\
& \dot{u}_{2}=-a u_{2}-u_{2}^{k} .
\end{aligned}
$$

Choose the following candidate Lyapunov function:

$$
V_{1}=\frac{1}{2}\left(u_{1}^{2}+u_{2}^{2}\right)
$$

The derivative of $V_{1}$ along the trajectory of (18) is

$$
\begin{aligned}
\dot{V}_{1} & =u_{1} \dot{u}_{1}+u_{2} \dot{u}_{2} \\
& =u_{1}\left(-a u_{1}-u_{1}^{k}\right)+u_{2}\left(-a u_{2}-u_{2}^{k}\right) \\
& \leq-u_{1}^{k+1}-u_{2}^{k+1}
\end{aligned}
$$

$$
\begin{aligned}
& =-\left(\frac{1}{2}\right)^{-(k+1) / 2}\left[\left(\frac{1}{2} u_{1}^{2}\right)^{(k+1) / 2}+\left(\frac{1}{2} u_{2}^{2}\right)^{(k+1) / 2}\right] \\
& \leq-\left(\frac{1}{2}\right)^{-(k+1) / 2}\left(\frac{1}{2} u_{1}^{2}+\frac{1}{2} u_{2}^{2}\right)^{(k+1) / 2} \\
& =-\left(\frac{1}{2}\right)^{-(k+1) / 2} V_{1}^{(k+1) / 2} .
\end{aligned}
$$

From Lemma 2, system (18) is finite-time stable. That means that there is a $T_{1}>0$ such that $u_{1} \equiv 0$ and $u_{2} \equiv 0$, for any $t \geq T_{1}$.

When $t>T_{1}$, the last three parts of system (11) become

$$
\begin{aligned}
& \dot{u}_{3}=-u_{3}+\mu_{3}, \\
& \dot{u}_{4}=-u_{4}+\mu_{4}, \\
& \dot{u}_{5}=-u_{5}+\mu_{5} .
\end{aligned}
$$



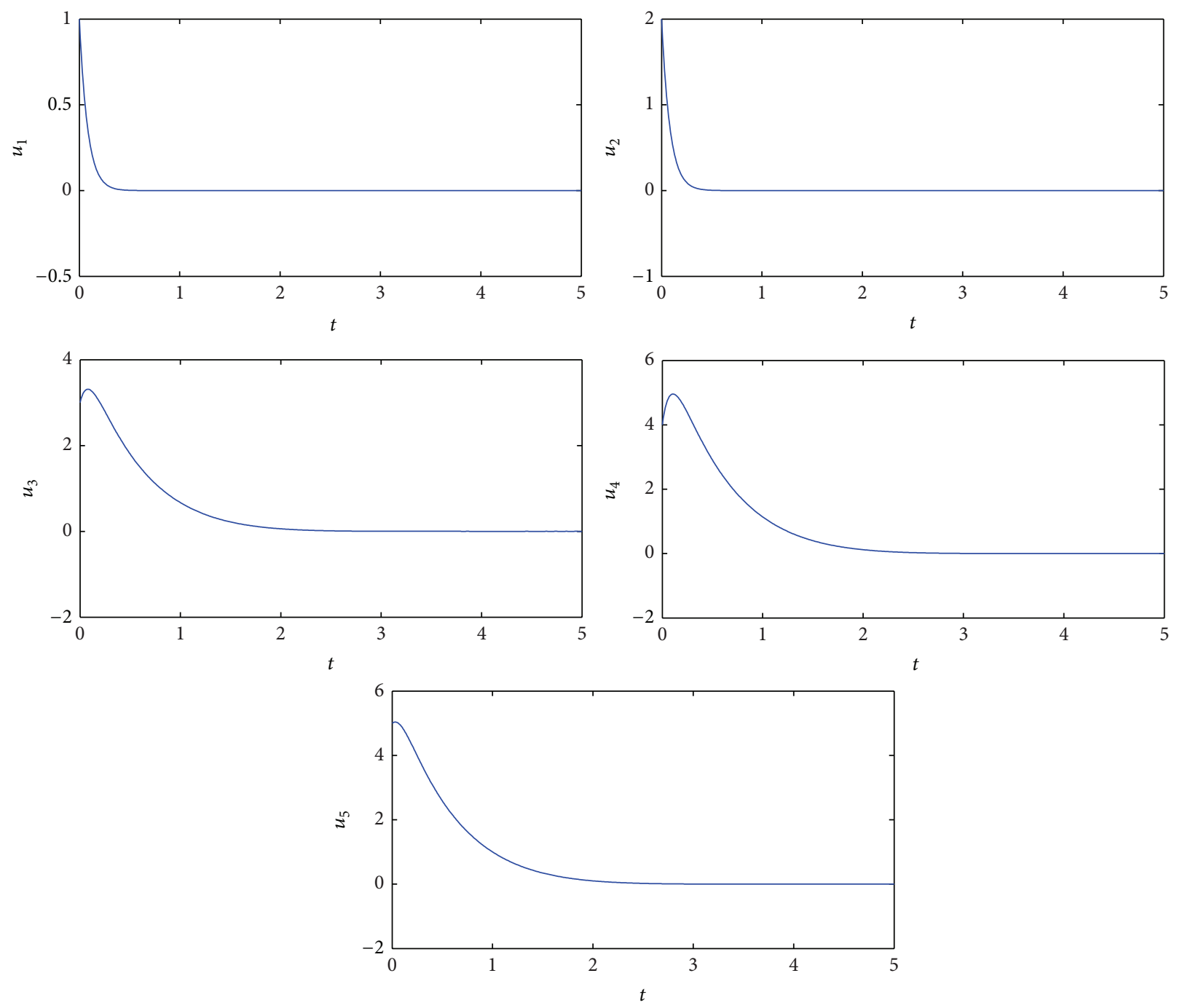

FIGURE 2: The states of the controlled system (11) with controllers (17).

Choose the following Lyapunov function for system (21):

$$
V_{2}=\frac{1}{2}\left(u_{3}^{2}+u_{4}^{2}+u_{5}^{2}\right) \text {. }
$$

The derivative of $V_{2}$ along the trajectories of (21) is

$$
\begin{aligned}
\dot{V}_{2}= & u_{3} \dot{u}_{3}+u_{4} \dot{u}_{4}+u_{5} \dot{u}_{5} \\
= & u_{3}\left(-u_{3}+\mu_{3}\right)+u_{4}\left(-u_{4}+\mu_{4}\right) \\
& +u_{5}\left(-u_{5}+\mu_{5}\right) .
\end{aligned}
$$

Substituting the controllers $\mu_{3}, \mu_{4}, \mu_{5}$ in (17) into the above equation yields

$$
\begin{aligned}
\dot{V}_{2}= & u_{3}\left(-u_{3}-u_{3}^{k}\right)+u_{4}\left(-u_{4}-u_{4}^{k}\right) \\
& +u_{5}\left(-u_{5}-u_{5}^{k}\right) \\
\leq & -u_{3}^{k+1}-u_{4}^{k+1}-u_{5}^{k+1}
\end{aligned}
$$

$$
\begin{aligned}
= & -\left(\frac{1}{2}\right)^{-(k+1) / 2} \\
& \times\left[\left(\frac{1}{2} u_{3}^{2}\right)^{(k+1) / 2}+\left(\frac{1}{2} u_{4}^{2}\right)^{(k+1) / 2}+\left(\frac{1}{2} u_{5}^{2}\right)^{(k+1) / 2}\right] \\
\leq & -\left(\frac{1}{2}\right)^{-(k+1) / 2}\left(\frac{1}{2} u_{3}^{2}+\frac{1}{2} u_{4}^{2}+\frac{1}{2} u_{5}^{2}\right)^{(k+1) / 2} \\
= & -\left(\frac{1}{2}\right)^{-(k+1) / 2} V_{2}^{(k+1) / 2} .
\end{aligned}
$$

Then from Lemma 2 , the states $u_{3}, u_{4}$, and $u_{5}$ will converge to zero at a finite time $T_{2}$. Then, after $T_{2}$, the states of system (11) will stay at zero; that is, the trajectories of the controlled system (11) converge to zero in a finite time.

Remark 6. Strategy 1 is easier to implement than Strategy 2, but the controllers (12) obtained by Strategy 1 are more complicated than the controllers (17) obtained by Strategy 
2. The controllers (17) are obtained by two steps. Simpler as they are, but the stabilization time with controllers (17) will be longer than that with controllers (12).

\section{Numerical Simulations}

In this section, two numerical examples are presented to illustrate the theoretical analysis. In the following numerical simulations, the fourth-order Runge-Kutta method is employed with time step size 0.001 . The system parameters are selected as $a=11$ and $b=20$, so that the complex PMSM system (3) exhibits chaotic behavior. The initial conditions for this system are given as $\left(z_{1}(0), z_{2}(0), z_{3}(0)\right)=(1+2 i, 3+4 i, 5)$; that is, $\left(u_{1}(0), u_{2}(0), u_{3}(0), u_{4}(0), u_{5}(0)\right)=(1,2,3,4,5)$.

Example 7. Consider Strategy 1 with the controllers (12). We choose $k=7 / 9$. Figure 1 shows the result of the numerical simulation. From Figure 1, we can see that it takes only a very short time to stabilize the controlled system (11) at zero. So system (11) achieves chaos control in a finite time.

Example 8. Consider Strategy 2 with the controllers (17). We still choose $k=7 / 9$. Figure 2 shows that the controlled system (11) achieves finite-time chaos control. From Figures 1 and 2, we can see that the stabilization time of the controlled system (11) in Figure 2 is longer than that in Figure 1.

\section{Conclusions}

Nowadays, the complex modeling of phenomena in nature and society has been the object of several investigations based on the methods originally developed in a physical context. In this paper, a complex PMSM system has been considered and the fast stabilization problem of this system has been investigated. Based on the finite-time stability theory, two kinds of simple and effective controllers for the complex PMSM system have been proposed to guarantee the global exponential stability of the controlled systems. During the past decades, the $H_{\infty}$ control strategy [24-26] has been widely celebrated for its robustness in counteracting uncertainty perturbations and external disturbances. Consequently, we will investigate the finite-time $H_{\infty}$ control problem of switched PMSM systems in our future work [2729].

\section{Conflict of Interests}

The authors declare that there is no conflict of interests regarding the publication of this paper.

\section{Acknowledgment}

This work was supported by the Natural Science Foundation of China under Grant nos. 11161055 and 61263042.

\section{References}

[1] J. P. Yu, J. W. Gao, Y. M. Ma, H. S. Yu, and S. F. Pan, "Robust adaptive fuzzy control of chaos in the permanent magnet synchronous motor," Discrete Dynamics in Nature and Society, vol. 2010, Article ID 269283, 13 pages, 2010.

[2] Z. Li, J. B. Park, Y. H. Joo, B. Zhang, and G. Chen, "Bifurcations and chaos in a permanent-magnet synchronous motor," IEEE Transaction on Circuits and Systems I, vol. 49, no. 3, pp. 383-387, 2002.

[3] Y. D. Son and G. H. Kang, "Drive system design for a permanent magnet motor with independent excitation winding for an electric bicycle," Journal of Electrical Engineering \& Technology, vol. 5, no. 4, pp. 623-630, 2010.

[4] V. Q. Leu, H. H. Choi, and J. W. Jung, "LMI-based sliding mode speed tracking control design for surface-mounted permanent magnet synchronous motors," Journal of Electrical Engineering \& Technology, vol. 7, no. 4, pp. 513-523, 2012.

[5] N. T. Vu, D. Y. Yu, H. H. Choi, and J. W. Jung, “T-S fuzzy-modelbased sliding-mode control for surface-mounted permanentmagnet synchronous motors considering uncertainties," IEEE Transactions on Industrial Electronics, vol. 60, no. 10, pp. 42814291, 2013.

[6] H. Zhang, Y. Shi, and A. Saadat Mehr, "Robust static output feedback control and remote PID design for networked motor systems," IEEE Transactions on Industrial Electronics, vol. 58, no. 12, pp. C5396-C5405, 2011.

[7] Q. S. Zhong, H. C. Li, H. Liu, and J. B. Yu, "A note on practical stability of nonlinear vibration systems with impulsive effects," Journal of Applied Mathematics, vol. 2012, Article ID 340450, 7 pages, 2012.

[8] H. Zhang, Y. Shi, and J. Wang, "On energy-to-peak filtering for nonuniformly sampled nonlinear systems: a markovian jump system approach," IEEE Transactions on Fuzzy Systems, vol. 22, no. 1, pp. 212-222, 2014.

[9] H. Ren and D. Liu, "Nonlinear feedback control of chaos in permanent magnet synchronous motor," IEEE Transactions on Circuits and Systems II: Express Briefs, vol. 53, no. 1, pp. 45-50, 2006.

[10] A. Loría, "Robust linear control of (chaotic) permanent-magnet synchronous motors with uncertainties," IEEE Transactions on Circuits and Systems. I. Regular Papers, vol. 56, no. 9, pp. 21092122, 2009.

[11] S.-C. Chang, "Synchronization and controlling chaos in a permanent magnet synchronous motor," Journal of Vibration and Control, vol. 16, no. 12, pp. 1881-1894, 2010.

[12] Y. Y. Hou, "Controlling chaos in permanent magnet synchronous motor control system via fuzzy guaranteed cost controller," Abstract and Applied Analysis, vol. 2012, Article ID 650863, 10 pages, 2012.

[13] H. H. Choi, "Adaptive control of a chaotic permanent magnet synchronous motor," Nonlinear Dynamics, vol. 69, no. 3, pp. 1311-1322, 2012.

[14] S. P. Bhat and D. S. Bernstein, "Finite-time stability of continuous autonomous systems," SIAM Journal on Control and Optimization, vol. 38, no. 3, pp. 751-766, 2000.

[15] D. Q. Wei and B. Zhang, "Controlling chaos in permanent magnet synchronous motor based on finite-time stability theory," Chinese Physics B, vol. 18, no. 4, pp. 1399-1403, 2009.

[16] A. C. Fowler, M. J. McGuinness, and J. D. Gibbon, “The complex Lorenz equations," Physica D, vol. 4, no. 2, pp. 139-163, 1982. 
[17] C. Z. Ning and H. Haken, "Detuned lasers and the complex Lorenz equations: subcritical and super-critical Hopf bifurcations," Physical Review A, vol. 41, no. 7, pp. 3826-3837, 1990.

[18] G. M. Mahmoud and T. Bountis, "The dynamics of systems of complex nonlinear oscillators: a review," International Journal of Bifurcation and Chaos in Applied Sciences and Engineering, vol. 14, no. 11, pp. 3821-3846, 2004.

[19] X. Y. Wang and H. C. Zhang, "Backstepping-based lag synchronization of a complex permanent magnet synchronous motor system," Chinese Physics B, vol. 22, no. 4, Article ID 048902, 2013.

[20] Z. Jing, C. Yu, and G. Chen, "Complex dynamics in a permanent-magnet synchronous motor model," Chaos, Solitons and Fractals, vol. 22, no. 4, pp. 831-848, 2004.

[21] S. P. Bhat and D. S. Bernstein, "Finite-time stability of homogeneous systems," in IEEE American Control Conference, Albuquerque, New Mexico, 1997.

[22] X. Y. Wang, X. L. Gao, and L. L. Wang, "Finite-time chaos synchronization of a new hyperchaotic Lorenz system," International Journal of Modern Physics B, vol. 27, no. 9, Article ID 1350033, 2013.

[23] X. Q. Huang, W. Lin, and B. Yang, "Global finite-time stabilization of a class of uncertain nonlinear systems," Automatica, vol. 41, no. 5, pp. 881-888, 2005.

[24] H. Zhang, Y. Shi, and A. Saadat Mehr, "On $H_{\infty}$ filtering for discrete-time takagi-sugeno fuzzy systems," IEEE Transactions on Fuzzy Systems, vol. 20, no. 2, pp. 396-401, 2012.

[25] H. Zhang, Y. Shi, and B. X. Mu, "Optimal H1-based linearquadratic regulator tracking control for discrete-time TakagiSugeno fuzzy systems with preview actions," Journal of Dynamic Systems, Measurement, and Control, vol. 135, no. 4, Article ID 044501, 5 pages, 2013.

[26] H. Zhang, J. Wang, and Y. Shi, "Robust $H_{\infty}$ sliding-mode control for Markovian jump systems subject to intermittent observations and partially known transition probabilities," Systems \& Control Letters, vol. 62, no. 12, pp. 1114-1124, 2013.

[27] D. Liberzon and A. S. Morse, "Basic problems in stability and design of switched systems," IEEE Control Systems, vol. 19, no. 5, pp. 59-70, 1999.

[28] B. Niu and J. Zhao, "Barrier Lyapunov functions for the output tracking control of constrained nonlinear switched systems," Systems \& Control Letters, vol. 62, no. 10, pp. 963-971, 2013.

[29] Q. S. Zhong, J. Cheng, and S. M. Zhong, "Finite-time $H_{\infty}$ control of a switched discrete-time system with average dwell time," Advances in Difference Equations, 2013. 


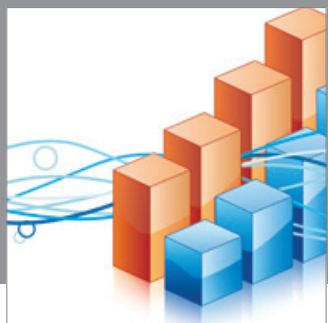

Advances in

Operations Research

mansans

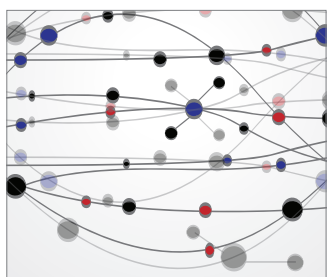

The Scientific World Journal
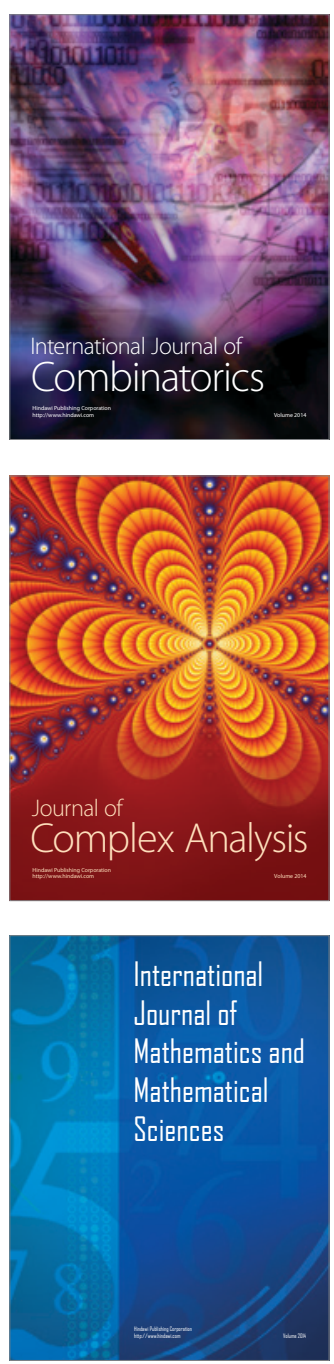
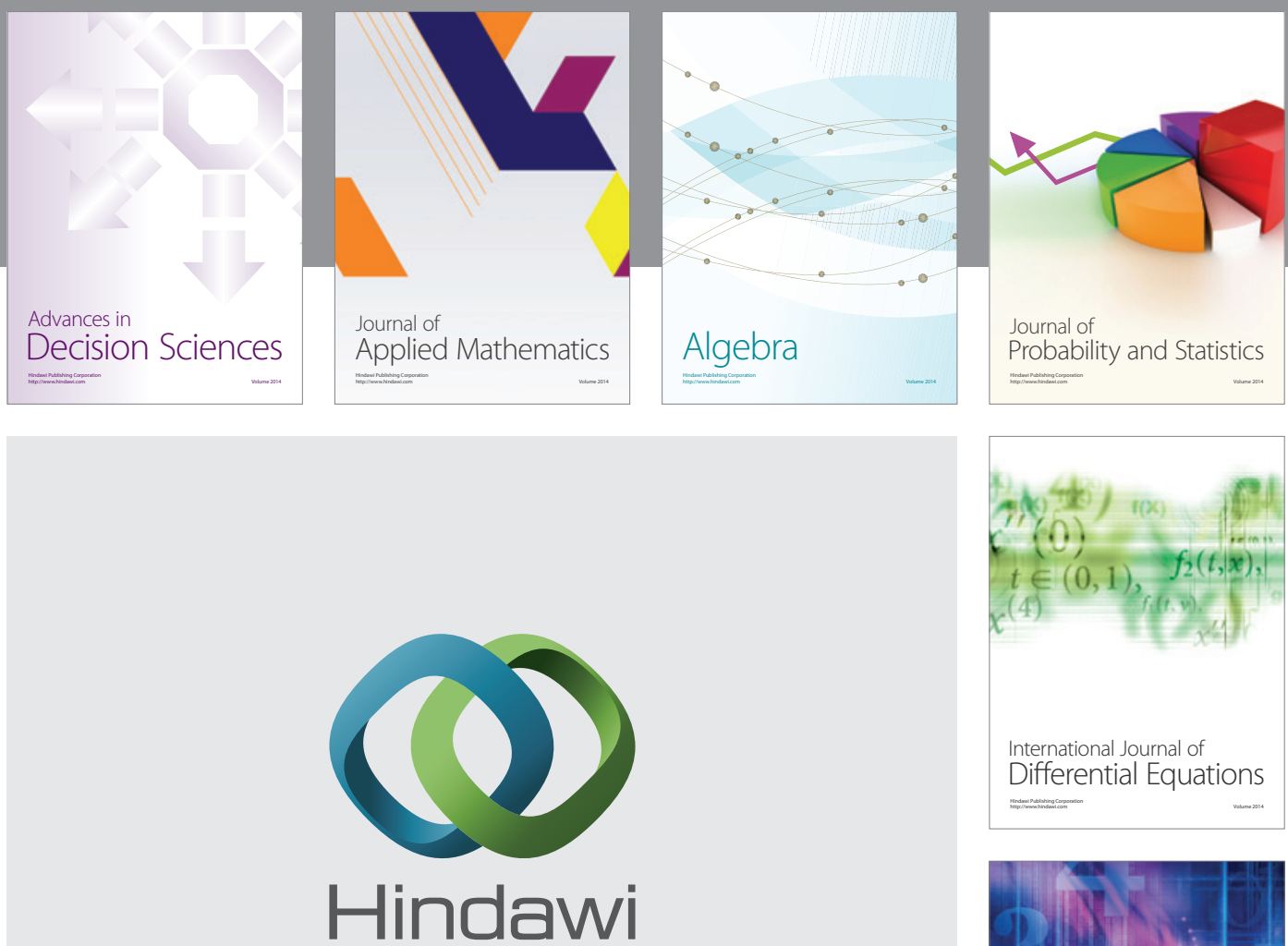

Submit your manuscripts at http://www.hindawi.com
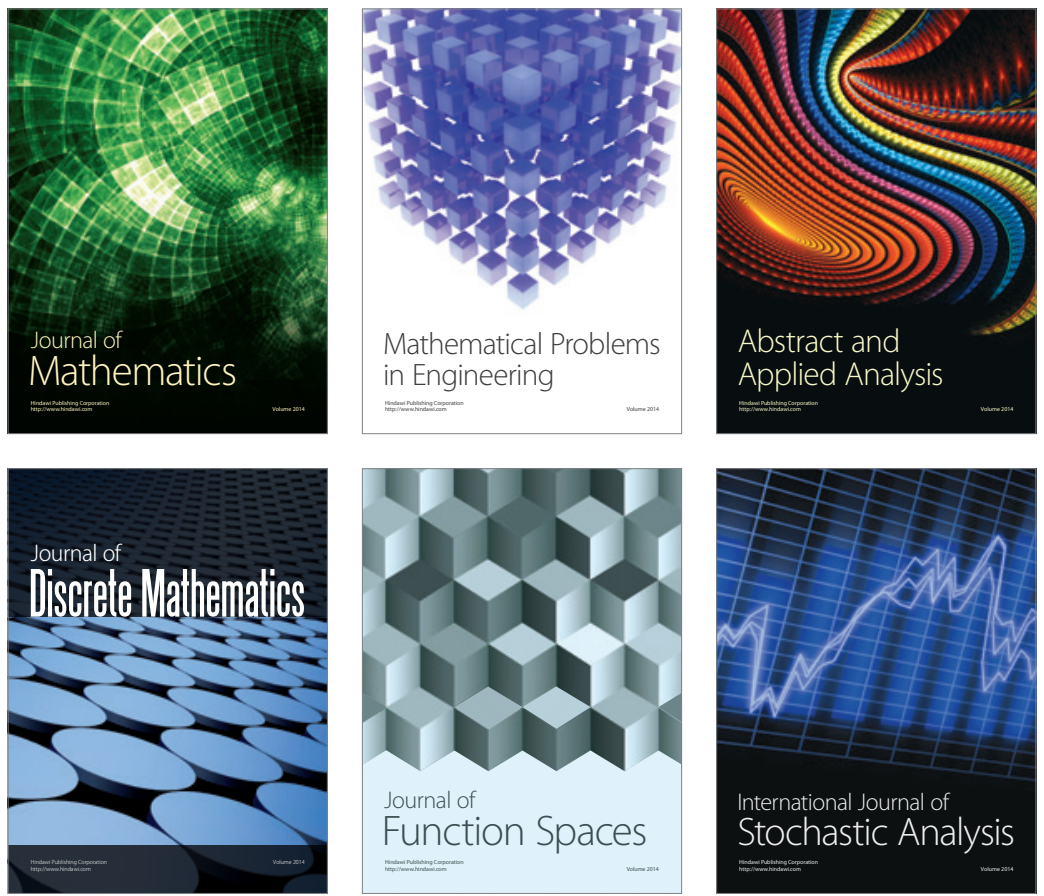

Journal of

Function Spaces

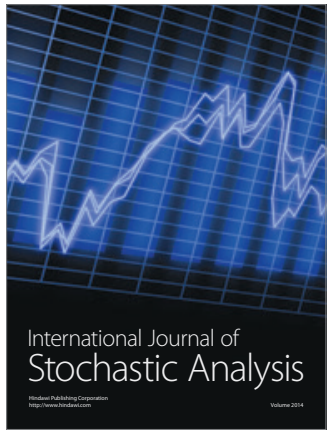

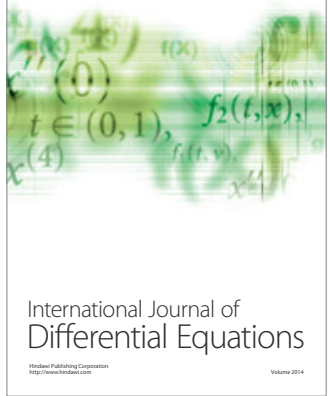
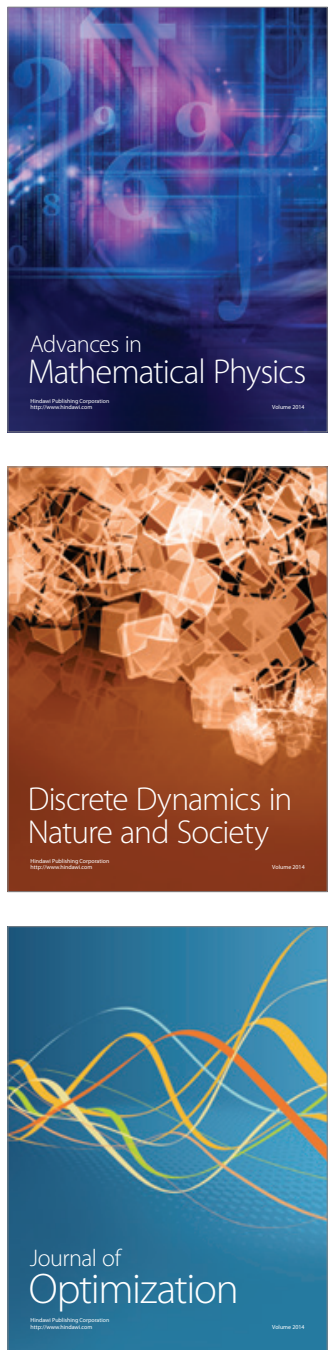